\title{
Anwendung der Ellipsometrie zur Untersuchung von Biomaterialien
}

Citation for published version (APA):

Hemker, H. C., Andree, H. A. M., \& Giesen, P. L. A. (1990). Anwendung der Ellipsometrie zur

Untersuchung von Biomaterialien. Hämostaseologie, 10, 71-76. https://doi.org/10.1055/S-0038-1655186

Document status and date:

Published: 01/01/1990

DOI:

10.1055/S-0038-1655186

Document Version:

Publisher's PDF, also known as Version of record

\section{Please check the document version of this publication:}

- A submitted manuscript is the version of the article upon submission and before peer-review. There can be important differences between the submitted version and the official published version of record.

People interested in the research are advised to contact the author for the final version of the publication, or visit the DOI to the publisher's website.

- The final author version and the galley proof are versions of the publication after peer review.

- The final published version features the final layout of the paper including the volume, issue and page numbers.

Link to publication

\footnotetext{
General rights rights.

- You may freely distribute the URL identifying the publication in the public portal. please follow below link for the End User Agreement:

www.umlib.nl/taverne-license

Take down policy

If you believe that this document breaches copyright please contact us at:

repository@maastrichtuniversity.nl

providing details and we will investigate your claim.
}

Copyright and moral rights for the publications made accessible in the public portal are retained by the authors and/or other copyright owners and it is a condition of accessing publications that users recognise and abide by the legal requirements associated with these

- Users may download and print one copy of any publication from the public portal for the purpose of private study or research.

- You may not further distribute the material or use it for any profit-making activity or commercial gain

If the publication is distributed under the terms of Article $25 \mathrm{fa}$ of the Dutch Copyright Act, indicated by the "Taverne" license above, 


\title{
Anwendung der Ellipsometrie zur Untersuchung von Biomaterialien
}

\author{
H. C. Hemker, H. A. M. Andree, P. L. A. Giesen
}

Abteilung für Biochemie und Institut für kardiovaskuläre Erkrankungen, Universität Limburg, Maastricht (Niederlande)

\section{Zusammenfassung}

Im folgenden wird ein Überblick über die dynamische Ellipsometrie gegeben. Diese Meßtechnik wurde in unserem Labor entwickelt und ermöglicht es, die an eine Oberfläche adsorbierte Masse einer dünnen Schicht aus organischem Material kontinuierlich zu messen. Voraussetzung für die Anwendung dieser optischen Technik ist eine reflektierende Oberfläche. Auf dieser Fläche können mehrere Schichten übereinander »gestapelt« werden. Die Methode ist so sensitiv, daß einschichtige Lagen von Proteinen und Fetten leicht gemessen werden können. Genaue Messungen innerhalb kurzer Zeitfolgen $(5-10 \mathrm{~s})$ sind möglich.

\section{Summary}

An overview is given of the technique of dynamic ellipsometry, a method developed in our laboratory that allows continuous measurement of the mass of thin layers of organic material adsorbed at interfaces. It is an optical technique that needs a reflecting surface as a basis. On this, multiple layers may be stacked. The sensitivity is such that monomolecular layers of proteins and lipids are easily measured. The method allows exact measurements at short $(5-10 \mathrm{~s})$ intervals.

\section{Einführung}

Die Ellipsometrie ist ein optisches Meßverfahren, mit dem Änderungen im Polarisationszustand des Lichtes
(Abb. 1) aufgezeichnet werden können, sobald Licht von einer Oberfläche reflektiert wird. Der Name dieses Verfahrens leitet sich von der Verwendung elliptisch polarisierten Lichts $\mathrm{ab}$ und daß mit diesem Verfahren die wesentlichen Charakteristika der Polarisation erfaßt werden, d.h. die Lage der Hauptachse in bezug zu der reflektierenden Oberfläche und die Elliptizität. Anders als jene Methoden, die mit der Lichtabsorption oder Streuung arbeiten, ist es nun möglich, Dimensionen, die kleiner als die Wellenlänge des angewendeten Lichts sind, zu erfassen. Daher ist es mit dieser Methode auch möglich, Eigenschaften von monomolekularen Schichten, die an der Interface adsorbiert werden, zu bestimmen. So ist diese Methode besonders geeignet, die Adsorption von Proteinen zu messen. Da die Adsorption von Proteinen immer der erste Schritt ist, der über die (In-)Kompatibilität eines Materials entscheidet, ist die Ellipsometrie möglicherweise ein überaus nützliches Verfahren für die Entwicklung von Biomaterialien.

\section{Skizzierung der Technik}

Zuerst ist es notwendig, sich die wichtigsten Eigenschaften von polarisiertem Licht ins Gedächtnis zu rufen (Abb. 1). Jeder kennt das Konzept des planen polarisierten Lichts: Senkrecht zu der Richtung des einfallenden Lichts wird dieser Polarisationstyp als Linie projiziert. Sobald dieses Licht auf eine reflektierende Oberfläche trifft, wird es als elliptisch polarisiertes Licht zurückgeworfen. Dies ist gleichsam als Resultat von zwei planen pola- risierten Strahlen vorstellbar, deren Polarisationsebenen im rechten Winkel zueinanderstehen und eine Phasendifferenz aufweisen. In der Ebene, die senkrecht zur Richtung der Fortbewegung des Lichts steht, wird dieses Licht als Ellipse dargestellt. Wenn elliptisch polarisiertes Licht auf eine reflektierende Oberfläche trifft, ändert sich die Elliptizität. Unter exakten Bedingungen kann sich diese Ellipsenform $\mathrm{zu}$ linear polarisiertem Licht reduzieren. Elliptisch polarisiertes Licht definierter Wellenlänge und Intensität ist weiterhin charakterisiert durch den Azimut $(\varnothing)$ und die Elliptizität $(\chi)$ der Ellipse. Ellipsometrie wird angewendet, um den Azimut und die Elliptizität zu bestimmen. Die wesentlichen Grundlagen der Ellipsometrie und des polarisierten Lichts können der angegebenen Literatur entnommen werden $(2,8)$.

In Abbildung 2 ist ein Ellipsometer abgebildet. Eine schematische Darstellung findet sich in Abbildung 3 . Das Ellipsometer besteht aus einer Lichtquelle (He-Ne Laser, Spectra Physics, Wellenlänge $=632,8 \mathrm{~nm}$ ), die in einer bestimmten Ebene polarisiertes Licht ausstrahlen kann. Die erste Polarisierung dieses Lichts findet an einem zirkulär polarisierenden Filter statt. Es entsteht zirkulär polarisiertes Licht. Hinter diesem Filter ist ein Prisma nachgeschaltet, das Licht in jeder erforderlichen Ebene polarisieren kann. Das Licht passiert den Kompensator, der eine Phasendifferenz von $1 / 4$ der Wellenlänge zwischen den Lichtkomponenten parallel und senkrecht zur der Richtung des einfallenden Lichts introduziert, und wird dadurch elliptisch polarisiert. Bei Reflexion von der Versuchsoberfläche 


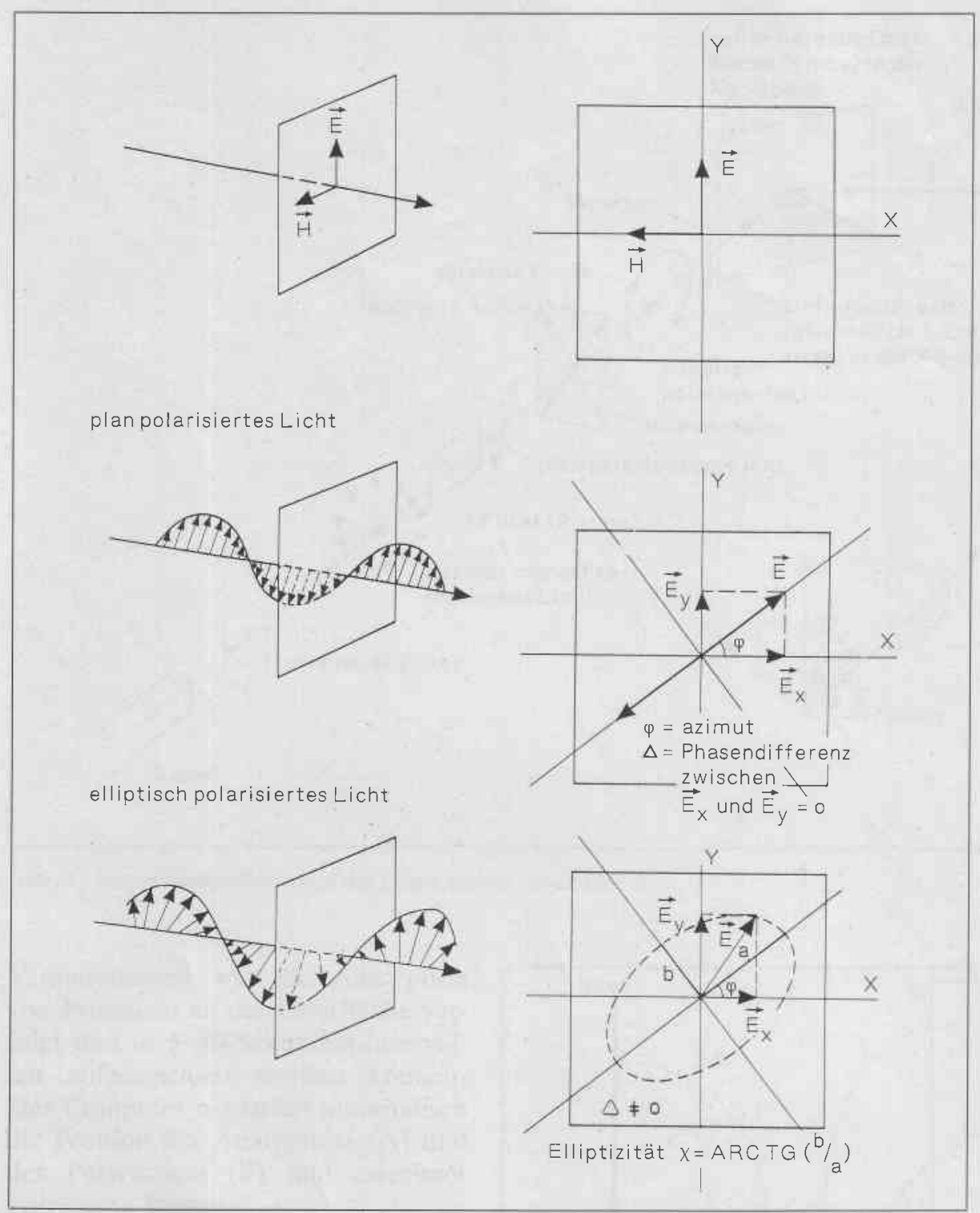

Abb. 1 Diagramm des polarisierten (plan and elliptisch) Lichts

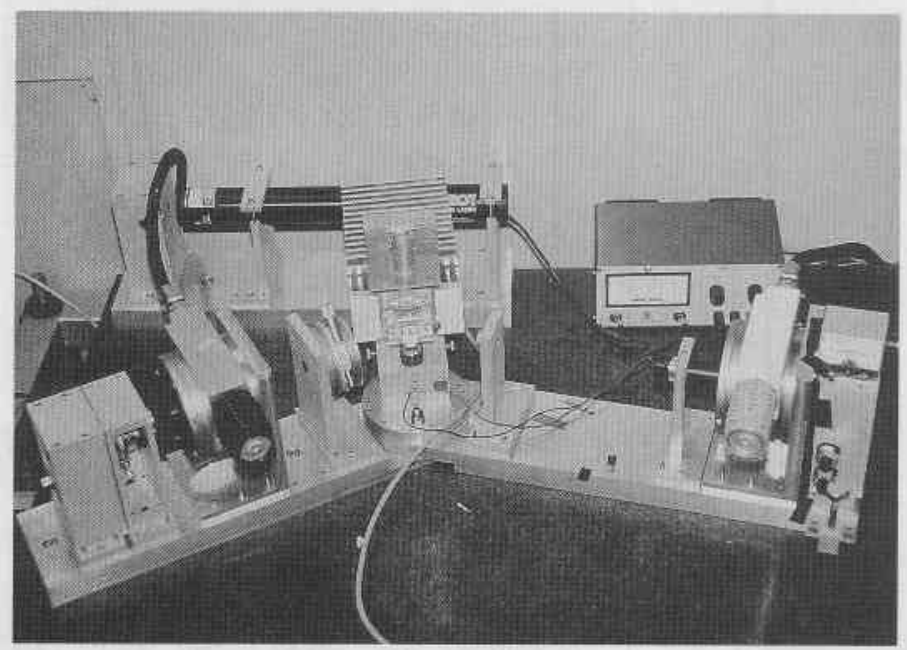

Abb. 2 Das Ellipsometer. Aus Platzgründen konnte der Laser im Hintergrund nicht in einer Linie mit der optischen Achse des Polarisators gebracht werden. Das Licht wird über einen Spiegel dorthin geleitet. Im Vordergrund sind zwei Aluminiumstützen zu sehen, dic auf einer Marmorplatte angebracht sind. Diese treffen in der Mitte in einem Winkel aufeinander, der durch die Reflexion des Lichts von der in die Küvette eintauchenden Oberfläche bestimmt wird. Weitere Einzelheiten siehe Text. verändert sich die Elliptizität. Auf einer bestimmten Polarisationsebene, die durch das erste Prisma, den Polarisator, festgelegt wird, wird das elliptisch polarisierte Licht als planes polarisiertes Licht zurückgeworfen. Die Ebene der Polarisation wird über ein zweites polarisiertes Prisma, den Analysator, gemessen. Dieses ist das letzte Element vor der Photodiode, mit der die Intensität des austretenden Lichts gemessen wird. Die beiden sich drehenden Prismen werden durch $»$ stepping motors « positioniert; diese wiederum werden durch ein Computerprogramm gesteuert, und zwar so, daß die Intensität des Lichts an der Photodiode minimiert wird. Es ist wichtig, $\mathrm{da} ß$ der Polarisator so positioniert ist, daß eine plane Polarisierung des reflektierten Lichts stattfindet und damit der Analysator die Ebene der Polarisation erfassen kann. Aus den Positionen des Polarisators und des Analysators lassen sich die entsprechenden Parameter einer Veränderung der Elliptizität im Rahmen der Reflexion errechnen. Solange das Licht an einer Oberfläche ohne dielektrische Eigenschaften reflektiert wird, bestimmt der komplexe Refraktionsindex der Oberfläche die Änderung der Elliptizität. Sobald an der Oberfläche eine dünne dielektrische, transparente Schicht adsorbiert worden ist, wird die Elliptizität $(\chi)$ auch verändert. Sie wird dann durch die Dicke (d) und den Refraktionsindex (n) dieser Schicht bestimmt. Geeignete Oberflächen unter optischen Gesichtspunkten sind z. B. Chrom oder Silizium, da sie einen hohen Refraktionsindex und einen hohen Reflexionskoeffizienten haben. Andererseits sind ihre Eigenschaften als Proteinabsorbierer nicht notwendigerweise für die biologischen Zwecke relevant. Ellipsometrie erlaubt es jedoch, daß verschiedene dielektrische Schichten gleichsam übereinander »gestapelt « werden und dann getrennt bestimmt werden können. Von daher ist es auch möglich, viele PhospholipidBilayers auf ein reflektierendes Siliziumkristall zu legen und die Bindung von Proteinen an diesen Schichten zu untersuchen.

In unserem Labor konnte die Positionierung des Polarisators und Analysators automatisiert werden, so daß 


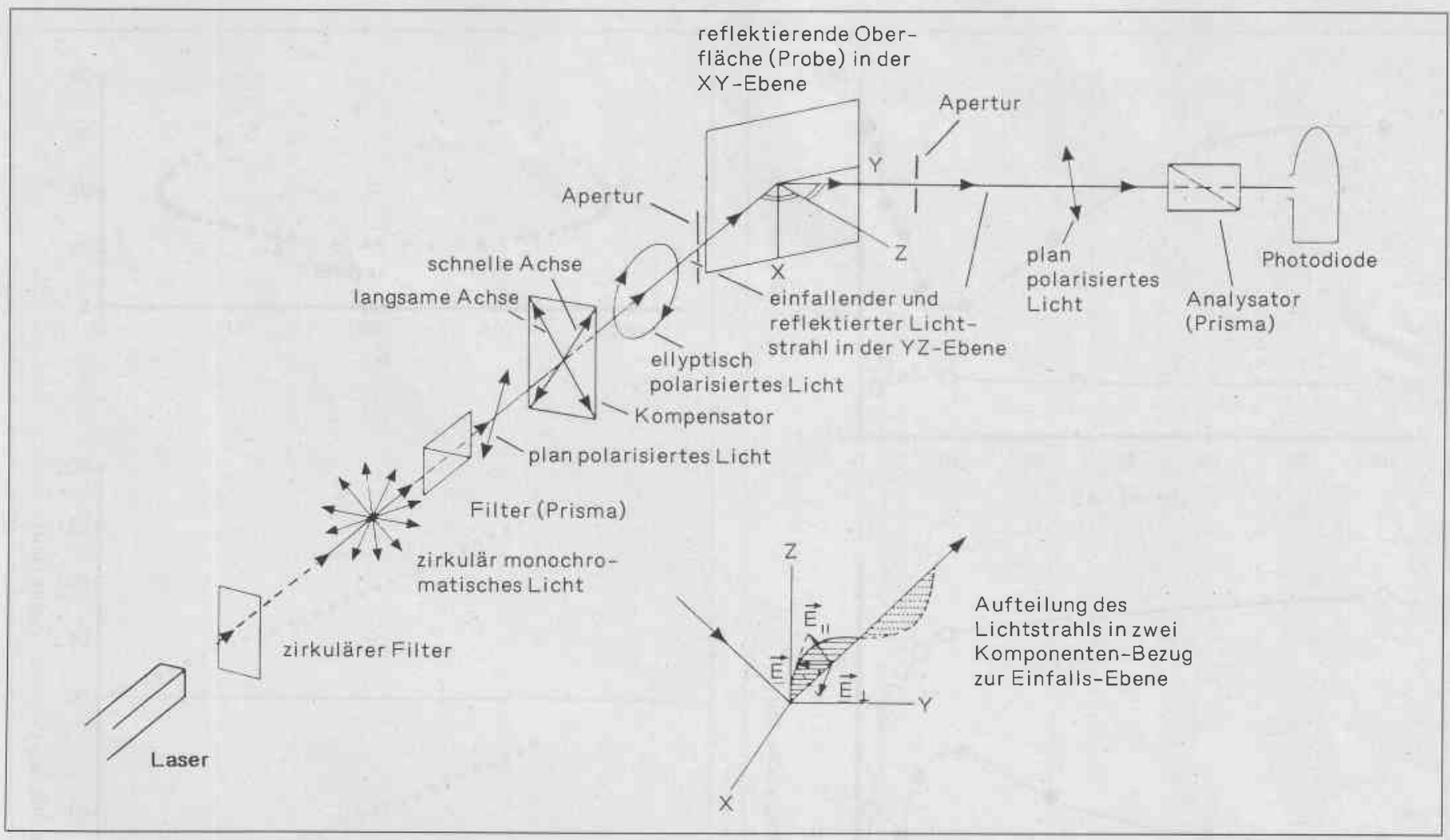

Abb. 3 Schematisches Schaubild des Ellipsometers. (Aus Ref. 3)

Veränderungen wie die Adsorption von Proteinen an der Oberfläche verfolgt und in 5-10-Sekunden-Intervallen aufgezeichnet werden können. Der Computer registriert automatisch die Position des Analysators (A) und des Polarisators (P) und errechnet korrigierte Werte:

$$
\Delta(=1 / 2 \pi+2 p), \Psi(=a)(2,4,8) .
$$

Es besteht eine komplizierte Beziehung zwischen $\Delta$ und $\Psi$ sowie der Dicke und dem Refraktionsindex. In Abbildung 4 ist diese Beziehung für ein System berechnet, das aus einer reflektierenden Siliziumoberfläche von Schichten mit unterschiedlichen Refraktionsindices und unterschiedlicher Dicke besteht. Der Refraktionsindex schwankt zwischen 1.1 und 3.5. Jede Linie repräsentiert $25 \AA$ Serienschritte bei einem festen Refraktionsindex. Dieser Graph zeigt die Unterschiede der Sensitivität von Analysator und Polarisator in verschiedenen Regionen der Ebene. Im Gebiet A $\mathrm{z}$. B. ist $\Delta$ nur eine Funktion von $\mathrm{d}$ und $\Psi$ von $\mathrm{n}$; im Gebiet B trifft das Gegenteil zu. Im Gebiet $C$ ist $\Psi$ eine Funktion von $\mathrm{d}$ und $\Delta$ reagiert nicht sehr empfindlich auf $\mathrm{n}$. Im Gebiet D

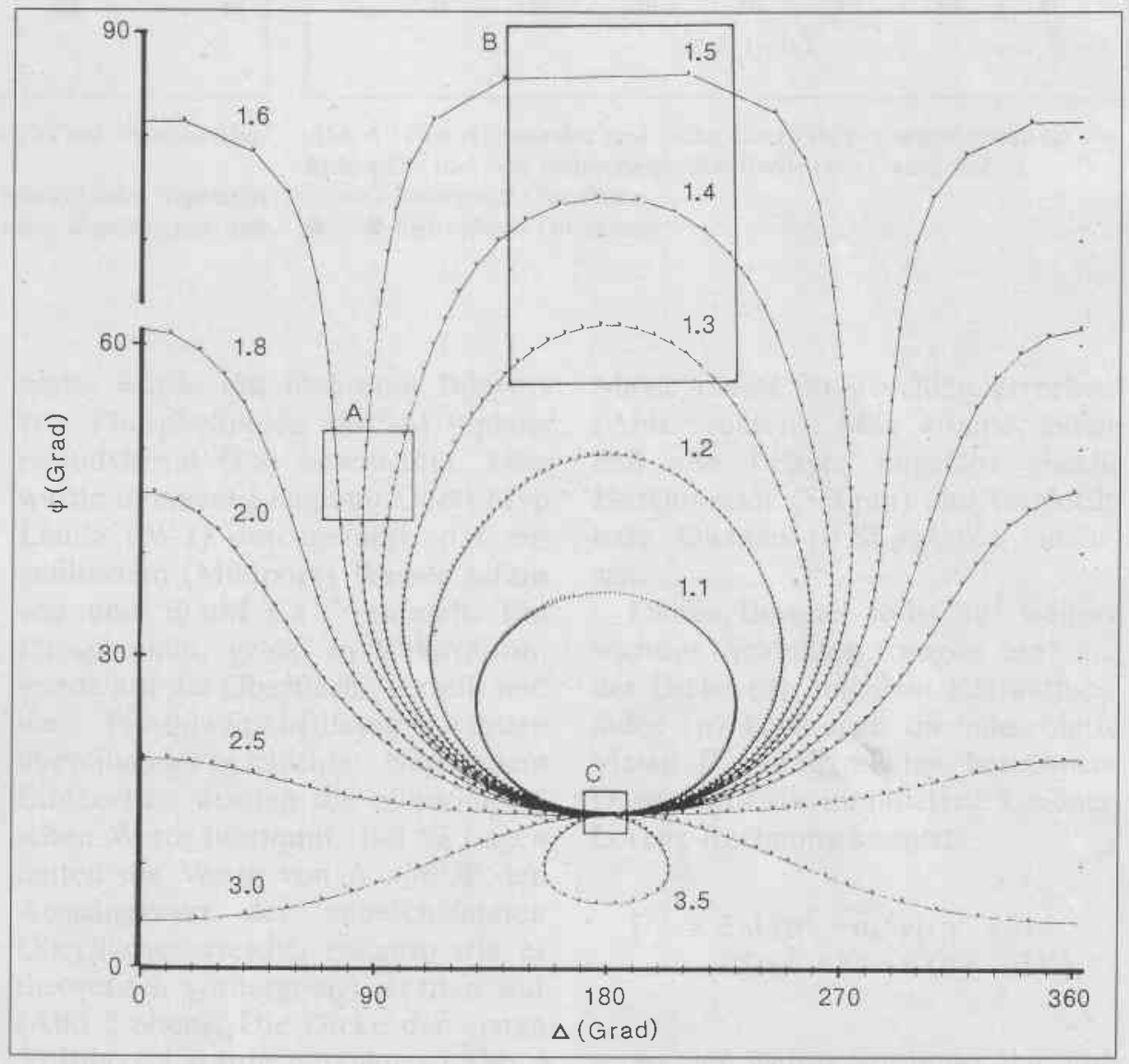

$A b b, 4$ Darstellung von $\Delta, \Psi$ auf $\mathrm{n}, \mathrm{d}$ für eine Siliziumoberfläche

Winkel des Auftretens $68^{\circ}$, Refraktionsindex des Mediums 1, (Luft) Wellenlänge 632,8 nM. (aus Ref. 3; s. Text) 


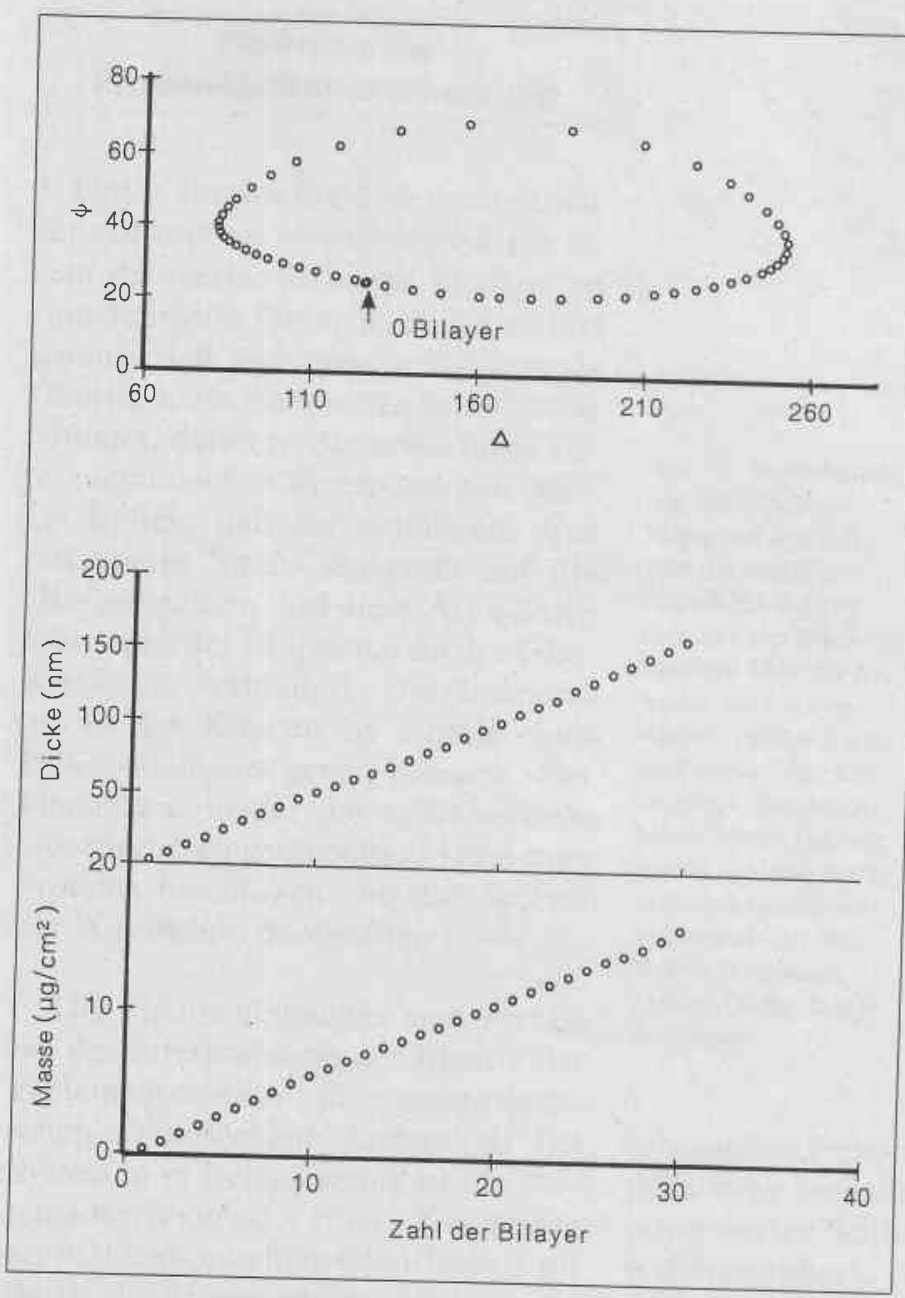
Abb.5 Ellipsometrische Messungen von 53 Bilayers auf Phosphatidyl-
serin

Oben: Werte von $\Delta$ und $\Psi$ der einzelnen Bilayer; Mitte: Dicke, abgelesen aus den Daten sub A; Unten: Masse, berechnet aus Durchmesser und Refraktionsindex.
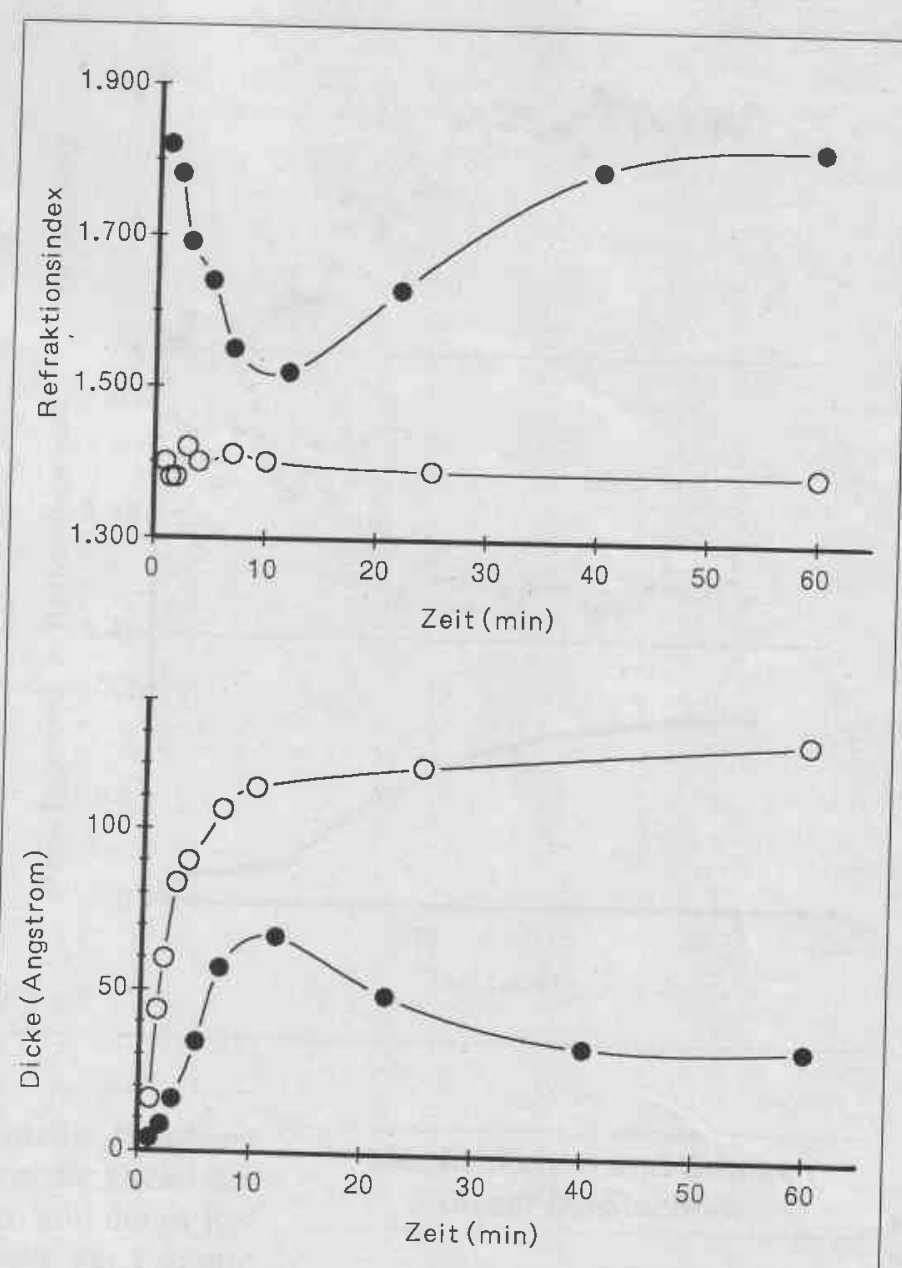

Abb. 6 Refraktionsindex und Dicke einer Fibrinogenadsorption an eine hydrophile und cine hydrophobe Oberfläche (mod. nach Ref. 3 ) O-O hydrophile Oberfläche

- hydrophobe Oberfläche verursacht eine Veränderung der Dikke von $25 \AA$ eine Veränderung von $\triangle$ von $70^{\circ}$, wohingegen in C die Sensitivität über $10 \mathrm{mal}$ geringer ist. Betrachtet man diese graphische Darstellung, so erkennt man, daß eine Simulation der Situation, die experimentell erwartet wird, sehr hilfreich sein kann, um herauszufinden, inwieweit die Versuchsbedingungen angemessen sind oder wie man sie adäquat gestalten kann. Falls für den Bereich einer bestimmten $\mathrm{zu}$ erwartenden Dicke und eines zu erwartenden Refraktionsindex unzureichende Sensitivität resultiert, kann eine Veränderung der Wellenlänge oder des Einfallwinkels oder der reflektierenden Oberfläche die Situation grundlegend verändern. Ein Anwendungsbeispiel dieser Theorie ist in Abbildung $5 \mathrm{zu}$ sehen. Eine hydrophobe (Chromcoating) Glas- platte wurde mit mehreren Bilayers von Phospholipiden di-C-14:0-phosphatidylserin (PS) beschichtet. Dies wurde in einem Langmuir-Gerät (Typ Lauda FW-1) durchgeführt, das mit gefiltertem (Millipore) Wasser gefüllt war und $50 \mu \mathrm{M} \mathrm{Ca}^{++}$enthielt. Ein Phospholipid, gelöst in Chloroform, wurde auf die Oberfläche verteilt und die Phospholipid-Bilayer wurden übereinander geschichtet. Nach jedem Eintauchen wurden die ellipsometrischen Werte bestimmt. Bei 53 Lagen hatten die Werte von $\Delta$ und $\Psi$ den Ausgangswert der unbeschichteten Oberfläche erreicht, genauso wie es theoretisch vorhergesagt worden war (Abb. 5 oben). Die Dicke der ersten 30 Bilayers wurde berechnet (Abb. 5 Mitte). Ebenso wurde aus dem Durchmesser und dem Refraktionsindex (Range 1.45-1.61) die absorbierte
Masse dieser 30 Schichten errechnet (Abb. 5 unten). Man konnte sehen, daß alle Bilayer ungefähr gleiche Durchmesser $(5,3 \mathrm{~nm})$ und vergleichbare Dichten $\left(0,54 \mu \mathrm{g} / \mathrm{cm}^{2}\right)$ aufwiesen.

Dieses Beispiel weist auf weitere wichtige Schlußfolgerungen hin: aus der Dicke (d) und dem Refraktionsindex (n) kann man die adsorbierte Masse $\Gamma$ von Proteinen berechnen. Dazu wurde die modifizierte LorentzLorenz-Rechnung benutzt:

$$
\begin{aligned}
\Gamma= & 3 \mathrm{~d}\left(\mathrm{n}^{2}-\mathrm{n}_{\mathrm{b}}^{2}\right) /\left[\left(\mathrm{n}^{2}+2\right) .\right. \\
& \left.\left.\cdot\left(\mathrm{r}^{2} \mathrm{n}_{\mathrm{b}}^{2}+2\right)-\mathrm{v}\left(\mathrm{n}_{\mathrm{b}}^{2}-1\right)\right)\right],
\end{aligned}
$$

$\mathrm{n}_{\mathrm{b}}$ ist der Refraktionsindex des Puffers, $r$ ist die spezifische molare Refraktivität $(\mathrm{ml} / \mathrm{g})$; und $\mathrm{v}$ ist das partielle spezifische Volumen $(\mathrm{ml} / \mathrm{g})(5,6)$. 


\section{Die Praxis der \\ Proteinadsorptionsmessung}

Unser Interesse gilt in erster Linie der Adsorption von Proteinen aus einem definierten flüssigen Medium an eine definierte Grenzfläche. Dies setzt voraus, $\mathrm{da} \beta$ sich eine reflektierende Oberfläche in einer speziellen Küvette befindet, deren rechte sowie linke Seite zueinander in einem solchen Winkel stehen, daß der einfallende und reflektierte Strahl senkrecht auf das Glas auftreffen. Auf diese Art werden Störungen der Ellipsizität an der Glasoberfläche verhindert. Die Temperatur in der Küvette ist mittels eines Peltier-Elements genau geregelt. Zur Flüssigkeit, in der die reflektierende Oberfläche eingetaucht ist, kann man Proteine hinzufügen und den Verlauf der Adsorption beobachten (5-7).

Die Fließbedingungen sind wichtig bei der Interpretation der Kinetik der Proteinadsorption an makroskopischen Oberflächen. Anders als bei Systemen in freier Lösung ist die Proteinadsorption oder Enzymkonversion an makroskopischen Oberflächen oft durch die Transportrate der Proteine zur Oberfläche hin begrenzt. Da die Ellipsometrie Proteinkinetiken an makroskopischen Oberflächen mißt, können auch dynamische Fließbedingungen studiert werden (3). Abbildung 6 zeigt die Adsorption von Fibrinogen an einer hydrophilen und einer hydrophoben Oberfläche. Es wird beobachtet, daß die Adsorption an einer hydrophilen Oberfläche nur eine Veränderung des Durchmessers der adsorbierten Schicht bringt, wohingegen die Adsorption an einer wasserabweisenden Oberfläche Veränderungen des Durchmessers und des Refraktionsindex hervorrufen. Die Erhöhung des Refraktionsindexes zusammen mit abnehmender Dicke der adsorbierten Schicht weisen auf eine Denaturierung des Fibrinogens hin, sobald es adsorbiert worden ist.

Die Veränderung dieses Durchmessers (d) und des Refraktionsindex (Abb. 6 oben) treten letztendlich ohne Veränderungen der Menge des adsorbierten Materials auf. Sind die Bedingungen weniger ideal, so treten häufig
Abb. 7 Bestimmung von adsorbicrter Masse aus den Angaben $n$ und $d$ Faktor-Xa-Adsorption an einer Phospholipid-Oberfläche wurde bei $t=0$ gestartet. Bei $t=5$ wur de Prothrombin hinzugefügt. Die adsorbierte Masse (unten) wurde aus dem beobachteten Durchmesser (oben) und dem Refraktionsindex (Mitte) (siehe Text) errechnet.

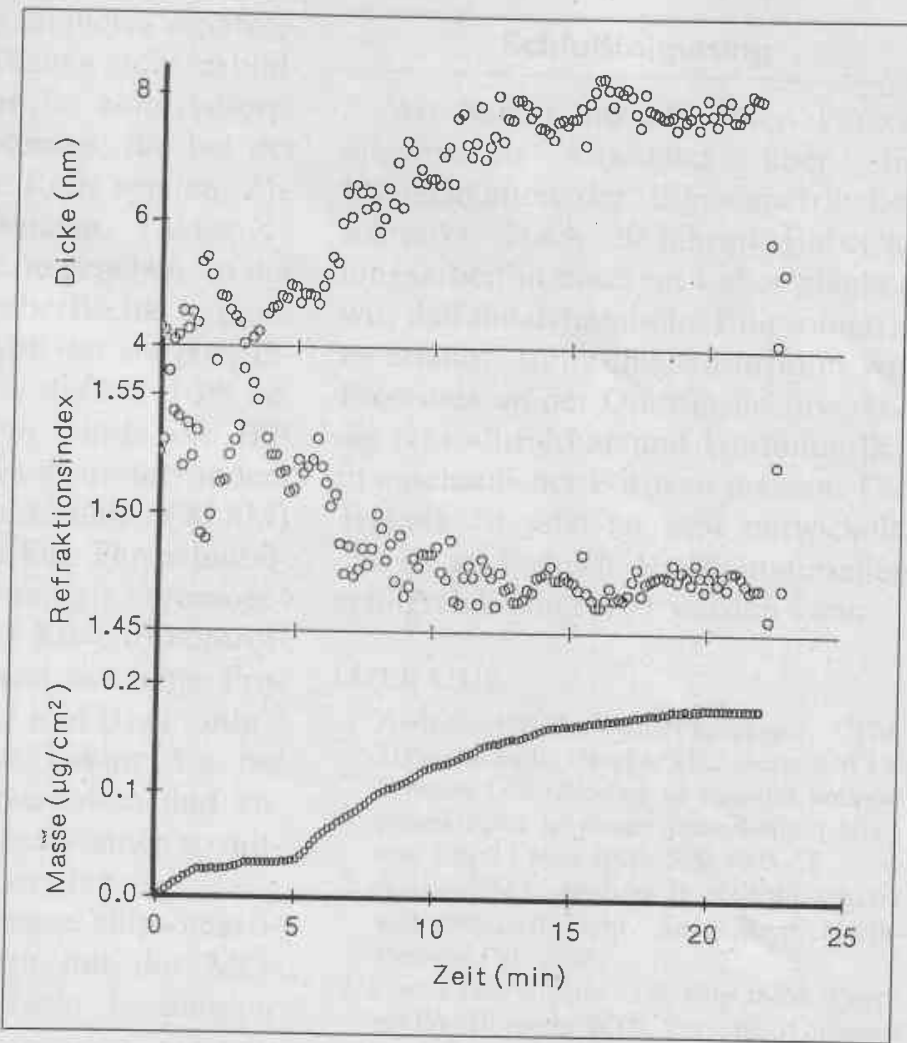

erhebliche experimentelle Probleme auf. Diese beeinflussen die Dicke der adsorbierten Schichten und deren Refraktionsindices. Sobald die gesamte adsorbierte Masse berechnet wird, stören diese Einflüsse nicht mehr (Abb. 7). Bei den meisten praktischen Anwendungen ist die adsorbierte Masse eines Proteins als Funktion der Zeit die wichtigste Variable. Im derzeitigen Versuchsstadium beträgt die Minimalmenge an Material, das auf einer Oberfläche nachgewiesen werden kann, $1 \mathrm{ng} / \mathrm{cm}^{2}$.

\section{Möglichkeiten und Grenzen dieser Meßtechnik}

Die Bedeutung dieser Technik wird durch den Umstand limitiert, daß, ähnlich wie bei einer Waage, die vorhandene Menge mit hoher Empfindlichkeit registriert wird, aber nichts darüber ausgesagt werden kann, welche Art von Molekülen adsorbiert wird. Die Tatsache, daß grundsätzlich die Zahl der übereinandergelagerten Schichten, die im Ellipsometer
Abb. 8 Adsorption von Annexin $\mathrm{V}$ und einem spezifischen Antikörper Bei $t=0$ setzte die Annexin-V-Adsorption ein. In weniger als 2 Min. war die Adsorption beendet, Bei $t=10$ wurde ein spezifischer Antikörper hinzugefügt, der auf dem Annexin V auflagerte.

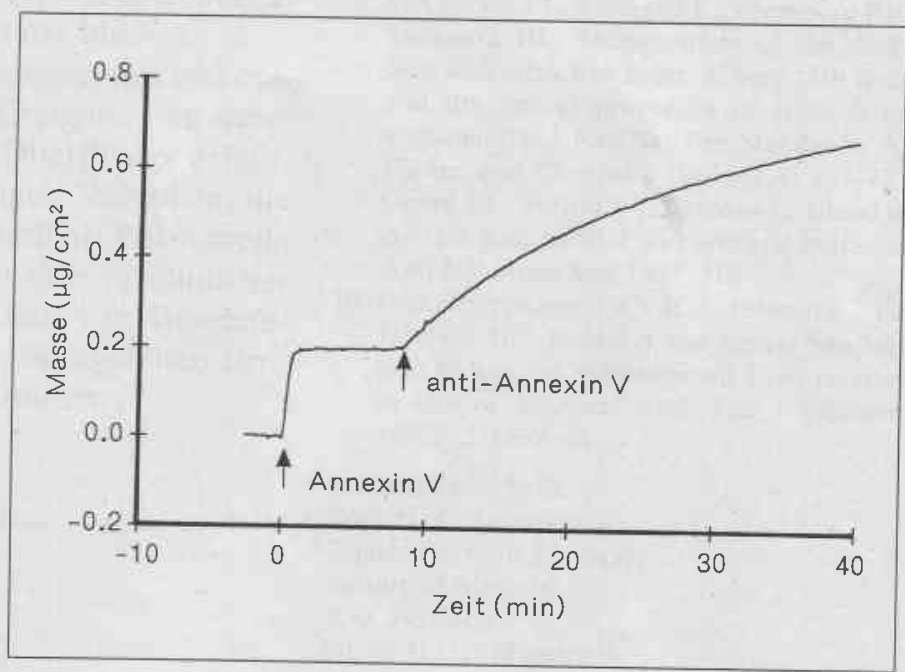


betrachtet werden können, unbegrenzt ist, führt dazu, daß die Messungen leicht ausgedehnt werden können, um immunologische Informationen über die Art der adsorbierten Proteine zu erhalten. Ist eine Oberfläche von einem bestimmten Protein bedeckt und wird ein spezifischer Antikörper hinzugefügt, so wird eine zweite Schicht an Proteinen entstehen, d. h., das Immunglobulin wird so adsorbiert, daß es auf der ersten Schicht aufliegt, sie gleichsam abdeckt. Abbildung 8 zeigt das Beispiel, wie ein Immunglobulin an der Proteinschicht adsorbiert wurde. Annexin V (das Gefäßantikoagulans $\alpha$ ) ist ein Protein, das sich an eine Phospholipid-Oberfläche bindet. Es verhindert die Blutgerinnung dadurch, daß es mit Gerinnungsfaktoren bei der Bindung an die prokoagulatorischen Phospholipidoberflächen konkurriert $(1,10)$. In Abbildung 8 ist dargestellt, wie Annexin $V$ von einem $20 \%$ die-C-18:1-PS/ $80 \%$ di-C-18:1-PC Bilayer aufgenommen wird. Die Adsorption bleibt stabil, sobald ein Sättigungspunkt an der Oberfläche erreicht ist. Dann wurde der Annexin-V-Antikörper hinzugegeben. Die Koadsorption des Antikörpers lag höher als $0,4 \mu \mathrm{g} / \mathrm{cm}^{2}$ und war damit deutlich höher als die Adsorption der Immunglobuline an einen einfachen Phospholipid-Bilayer $\left(<0,05 \mu \mathrm{g} / \mathrm{cm}^{2}\right)$.

Ein Problem tritt bei der Adsorption von Proteinen auf, die in einer komplexen Mischung mit Plasma enthalten sind, und zwar besonders dann, wenn die Oberfläche im Vergleich zum Volumen groß ist. In diesen Fällen kann die Adsorption nicht mit dem Langmuir-Ansatz auf der Grundlage von Konzentrationen und Dissoziationskonstanten beschrieben werden. Vroman zeigte als erster, daß Proteine im Plasma in einer bestimmten Reihenfolge adsorbiert und desorbiert werden. Das erste adsorbierte Protein wird durch ein anderes ersetzt usw., bis das endgültige Gleichgewicht erreicht ist. Solche Veränderungen können ohne zusätzliche Hilfsmittel nicht direkt mit der Ellipsometrie beobachtet werden. Sie erfordern zusätzliche Experimente, meist immunologischer Art (Überblick s. 9).

Es ist möglich, die biologische Aktivität der adsorbierten Proteine zu messen, falls ein geeignetes Analyseverfahren zur Verfügung steht. Abbildung 7 zeigt in der Tat eine Adsorption von zwei Proteinen, die bei der Blutgerinnung eine Rolle spielen. $\mathrm{Zu}$ erst wurde das Enzym, Faktor Xa (4 $\mathrm{nM}$ ) in die Küvette gegeben, in der sich eine Siliziumoberfläche befand, die mit einem Bilayer, der aus 80\% diC-18:1-PC und 20\% di-C-18:1-PS beschichtet war. Dann wurde die Bildung von Thrombin gestartet, indem das Substrat Prothrombin (50 nM) hinzugefügt wurde. Die Thrombinbildung konnte leicht mittels chromogener Substrate in der Küvette kontrolliert werden. Es fand sich eine Produktionsrate von $0,3 \mathrm{~mol}$ (II a) $\cdot \mathrm{min}^{-1}$ mol $(\mathrm{Xa})^{-1}$ ohne Faktor $\mathrm{Va}$ bei $20^{\circ} \mathrm{C}$. Adsorptionsverhalten und enzymatische Aktivitäten können so miteinander korreliert werden.

Ähnlich leicht können ellipsometrische Untersuchungen mit der Messung der Radioaktivität bestimmter Proteine verbunden werden, die vorher aber markiert worden sind (7)

\section{Spezifische Probleme bei Biomaterialien}

Die große Mehrzahl der Biomaterialien ist dielektrisch. Daher müssen geeignete reflektierende Oberflächen mit den Proteinen beschichtet werden, damit die Ablagerung des Proteins auf dem Material in angemessener Weise studiert werden kann. Dies kann problematisch sein, denn die Oberflächen müssen in einer Weise »gecoated « werden, daß ihre physiko-chemischen Eigenschaften für die Oberfläche des gesamten Untersuchungsmaterials weiterhin repräsentativ bleiben.

Da die Ellipsometrie mikroskopische, molekulare Ereignisse an einer makroskopischen Oberfläche erfaßt, ist sie eine der wenigen Techniken, die uns erlaubt, unmittelbar Phänomene zu beobachten, die sich in ähnlicher Weise an Oberflächen von Biomaterialien, also in einer biologischen Umgebung abspielen können.

\section{Abkürzungen:}

PS = Phospharidylserin

$\mathrm{PC}=$ Phosphatidylcholin

\section{Schlußfolgerung}

Wir haben uns auf einen kurzen allgemeinen Überblick über die Möglichkeiten der Ellipsometrie beschränkt. Nach 20 Jahren Entwicklungsarbeit in unserem Labor glauben wir, daß die dynamische Ellipsometrie es erlaubt, stetig die Adsorption von Proteinen an der Oberfläche zuverlässig reproduzierbar und kontinuierlich in anschaulicher Form zu messen. Die Technik ist jetzt so weit entwickelt, daß sie im Bereich der Biomaterialien erfolgreich eingesetzt werden kann.

\section{LITERATUR}

1. Andree HAM, Reutelingsperger CPM, Hauptmann R, Hemker HC, Hermes WTh, Willems GM. Binding of vascular anticoagulant alpha to planar phospholipid bilayers. J Biol Chem 1990; 265: 4923-28

2. Azzam RMA, Bashara N M. Ellipsometry and Polarized Light. Amsterdam: NorthHolland Publ, 1977

3. Corsel JW, Willems GW, Kop JMM, Cuypers PA, Hermens W'Th. The role of intrinsic binding rate in the adsorption of prothrombin, albumin and fibrinogen to phospholipid bilayers. J Colloid Interf Scie 1986; 111: $544-54$

4. Cuypers PA. Dynamic ellipsometry, biochemical and biomedical applications Thesis: University of Maastricht, 1976

5. Cuypers PA, Corsel JW, Janssen MP, Kop JMM, Hermens WTh, Hemker HC. The adsorption of prothrombin to phosphatidylserine multilayers quantitated by eliipsometry. J Biol Chem 1983; 258: 2426-31

6. Kop JMM, Cuypers PA, Lindhout $\mathrm{T}$, Hemker HC, Hermens WTh. The adsorption of prothrombin to phosphatidylserine monolayers quantitated by ellipsometry. J Biol Chem 1984; 259: 13993-98

7. Kop JMM, Willems GM, Hermans WTh. Binding of blood coagulation factor $\mathrm{V}$ to planar phospholipid double layers. J Colloid Interf Scie 1989; 133: 369-76

8. McCrackin FL, Passaglia E, Stromberg RR, Steinberg HL. Measurement of the thickness and refractive index of very thin films and the optical properties of surfaces by ellipsometry. J Res Nat Bur Standards; A, Physics and Chemistry 1963; $67 \mathrm{~A}: 363-77$

9. Onard EL, Turitto VT, Vroman L. Blood in contact with natural and artificial surfaces. Ann NY Acad Scie 1987, 516

10. Reutelingsperger CPM, Hornstra G, Hemker HC. Isolation and partial purification of a novel anticoagulant from arteries of human umbilical cord. Eur J Biochem 1985; 151: 625-29

Anschrift f, d. Verff:

Prof. H. C. Hemker

Rijksuniversiteit Limburg

Faculty of Medicine

P. O. Box 616

NL-6200 MD Maastricht 